\title{
LOS SUBSISTEMAS JURÍDICOS BRITÁNICOS (DERECHO CONSUETUDINARIO INGLÉS O COMMON LAW) Y EL SISTEMA JUDICIAL ESPAÑOL (CIVIL LAW)*
}

\author{
Francisco GodoY TENA**
}

SUMARIO: I. Introducción. II. Familias y sistemas de derecho. III. Derecho consuetudinario (common law) y derecho romano (civil law). IV. Origen del common law. V. Características del common law. VI. Derecho inglés y galés. VII. Origen y evolución del derecho inglés y galés. VIII. Derecho civil y derecho penal británico. IX. Origen y evolución del derecho escocés. X. Derecho civily derecho penal escocés. XI. Origen y evolución del derecho norirlandés. XII. Derecho español.

XIII. Derecho comparado. XIV. Conclusiones. XV. Bibliografia.

\section{INTRODUCGIÓN}

El ámbito del derecho constituye una pieza fundamental en la sociedad actual, en el cual podemos distinguir diversas familias y sistemas de derecho. Dentro de ellas podemos resaltar dos: por un lado, el civil law, derecho napoleónico cuyo origen se remonta al derecho romano. En este sistema nos centramos en el derecho español, su origen, evolución y explicación del mismo. Por otro, el common law, derecho consuetudinario o derecho inglés, claramente diferenciable del anterior. Originario en Inglaterra, el common law se extendió primeramente a Gales, Escocia e Irlanda del Norte y, en siglos sucesivos y de igual modo que en caso español, a otros países del mundo. Ante esta situación conviene por consiguiente detallar los ordenamientos jurídicos de Inglaterra y Gales, Escocia e Irlanda del Norte, todos ellos pertenecientes al sistema jurídico del Reino Unido.

* Artículo recibido el 7 de noviembre de 2017 y aceptado para su publicación el 13 de julio de 2018.

** ORCID: 0000-0001-6353-6900. Profesor del Grado de Traducción e Interpretación. Departamento de Filología Inglesa. Facultad de Filosofía y Letras de la Universidad Autónoma de Madrid. Correspondencia: Despacho 201-VIBIS, Avenida Tomás y Valiente, 1 Campus de Cantoblanco 28049, Madrid. Correo electrónico:f.godoy@uam.es.

Boletín Mexicano de Derecho Comparado, nueva serie, año LI, núm. 154, enero-abril de 2019, pp. 513-538. 
Ante esta distinción es necesaria realizar una comparativa entre ambos sistemas jurídicos a fin de poder establecer los parámetros comunes para facilitar a investigadores que realicen estudios de derecho comparado. Para ello, el presente estudio se inicia con las familias y sistemas de derecho para, posteriormente, realizar una explicación de ambos sistemas jurídicos por medio de una comparación descriptiva que recopile aspectos de ambos sistemas, claves para su comprensión.

\section{FAMILIAS Y SISTEMAS DE DERECHO}

Durante siglos se han podido constatar numerosos ordenamientos jurídicos en diversas partes del mundo. Glenn (2007) menciona acertadamente a los derechos judío, griego y egipcio que sobrevivieron al derecho romano, constatando la existencia de otros derechos bajo la dominación de Roma, ordenamientos clásicos a los que añadimos otros más modernos. Del mismo modo, Esmein (1905) describe las siguientes familias: Romanistic, Germanic, ${ }^{1}$ Anglo-Saxon, Slav and Islamic families (Esmein, 1905: 445).

En este sentido podemos sintetizar las ideas expuestas por Hertel (2009: 187), dentro de estas familias de derecho:

- Sistema jurídico de estados comunistas, incluidos dentro del civil law en su aspecto más general.

- Sistema jurídico nórdico, entre el common law y el civil law.

- Sistema jurídico islámico, ordenamiento único existente en numerosos países, existiendo un solapamiento en diversos países sudasiáticos entre el ordenamiento islámico y el common law.

- Sistema jurídico de Israel, en un principio basado en el common law durante la dominación británica, si bien es cierto que numerosas leyes están suplantando aquellas del common law por otras basadas en el derecho romano-germánico.

- Derecho de costumbres, propio de diversos países africanos, en donde se solapan el common law y el derecho de costumbres, dependiendo de las creencias religiosas de dichos países (Hertel, 2009: 187).

1 Familia de derecho de gran influencia para nuestro derecho, especialmente el administrativo.

Esta obra está bajo una Licencia Creative Commons

Atribución-NoComercial-SinDerivar 4.0 Internacional, IIJ-UNAM.

Boletín Mexicano de Derecho Comparado, núm. 154, enero-abril de 2019, pp. 513-538. 
En este punto son dignos de mención los países poscoloniales del continente africano, entre los que podemos citar, entre otros, Sudáfrica (con rasgos del Common Law), países poscoloniales de Portugal (Angola, Guinea-Bisáu y Cabo Verde) que continúan con el Código Civil portugués de 1966 o el Código Civil español de 1889, el cual continúa vigente en Guinea Ecuatorial.

Los diversos ordenamientos jurídicos comparten características comunes y divergentes a tener en cuenta, como es el caso del jurado, parte indiscutible de la mayoría de países anglófonos, como es el caso de Irlanda o Estados Unidos, por solo citar unos ejemplos. ${ }^{2}$ Sin embargo, en otros países el jurado no está presente, como es el caso de Israel o Sudáfrica, mientras que en el caso belga la figura del jurado fue abolida tras la ocupación francesa y retomada tras su independencia en 1831.

Stolze (2013) realiza una comparativa entre el denominado Common Law en los países anglosajones, que comentaremos posteriormente, y el derecho escrito en la mayoría de los países del continente europeo, derivado de la legislación constitucional romana, entre los que destacamos a los ordenamientos jurídicos español, francés o alemán.

\section{DERECHO GONSUETUDINARIO (COMMON LAW) Y DERECHO ROMANO (CIVIL LAW)}

La presente sección constituye el inicio de la comparativa de los dos sistemas a estudio: el common law y el civil law. El derecho consuetudinario presenta una serie de diferencias con el civil law o derecho romano a tener en consideración. Vázquez y del Árbol (2008: 15) realiza una distinción entre el derecho consuetudinario, a veces denominado "derecho común inglés", frente al derecho romano o continental, también conocido como derecho civil o napoleónico. La primera de las diferencias explicadas es la ausencia de una codificación escrita en el derecho consuetudinario o common law, frente a la presencia de documentos jurisprudenciales escritos, característica fundamental del derecho romano.

En segundo lugar, se puede mencionar que el fallo de "culpable" frente al "inocente/no culpable" difiere en parte en ambas familias del dere-

2 El ordenamiento jurídico español cuenta igualmente con jurado en determinados enjuiciamientos delictivos. 
cho. En el common law se atisba una posición de igualdad entre la acusación y la defensa, inexistente en el civil law, y que se puede observar en el plano lingüístico con el binomio "guilty", "non-guilty", propio de los países del common law, particularmente Inglaterra y Estados Unidos, frente a otros países continentales, más cercanos a modelo francés (civil law):

In favour of a list of questions or propositions presented to the jury... the Spanish judge prepares a verdict form or "objeto del veredicto" in the form of a list of propositions, some designated as favourable to the defendant, some as unfavourable, and the jury must decide whether they were proved during the trial (Doran, Sean y Jackson, 2000: 55).

\section{ORIGEN DEL COMMON LAW}

La importancia del derecho romano se refleja en el propio concepto de "common law", puesto que originariamente proviene de (ius commune). El Oxford Dictionary of Law define el concepto del siguiente modo:

The part of English law based on rules developed by the royal courts during the first three centuries after the Norman Conquest (1066) as a system applicable to the whole country, as opposed to local customs. The Normans... were mainly concerned with establishing a strong central administration and safeguarding the royal revenues... (Oxford University, 2015: 122).

El origen histórico se puede considerar a partir de la invasión normanda en 1066, puesto que el territorio se encontraba regulado por un sistema judicial muy poco desarrollado y homogéneo, siendo a partir de ese momento el germen jurídico del common law, y que se desarrollaría a partir de los siglos XII y XIII.

Los primeros movimientos del common law vinieron de la mano de jueces itinerantes, y en donde se decidía por medio de una comparación analógica entre los diferentes casos a examen. Los anteriores "sistemas jurídicos" existentes antes del siglo XII fueron, por tanto, reemplazados por este "derecho consuetudinario", común a aquellos pertenecientes a la corona inglesa.

La posterior colonización de diversas tierras hizo posible la extensión del common law a otros territorios que, tras independizarse de la corona in-

Esta obra está bajo una Licencia Creative Commons

Atribución-NoComercial-SinDerivar 4.0 Internacional, IIJ-UNAM.

Boletín Mexicano de Derecho Comparado, núm. 154, enero-abril de 2019, pp. 513-538. 
glesa, han mantenido el sistema jurídico del common law hasta la actualidad. Es por ello que el common law pasa a formar parte de los tres subsistemas que forman el sistema jurídico británico: inglés y galés, escocés y norirlandés.

\section{Garacterísticas DeL COMMON LAW}

Primeramente, consideremos las siguientes características propias del common law:

\section{Fuentes del common law}

En primer lugar, consideremos las diferentes fuentes del common law originado en Inglaterra, las cuales son las siguientes:

- Los repertorios de jurisprudencia (law reports, statute/statutory reports).

- Costumbres generales y particulares del reino, así como la costumbre que se ha llegado a adoptar dentro de los tribunales. Se denomina case law y es desarrollada por los magistrates, judges y los lords y ladies. Fuentes originales e históricas del derecho, como son los escritos de Bracton y Coke, entre otros.

- Equity (equidad). Mandamientos, deberes y obligaciones. Parte del derecho natural.

Por otra parte, podemos asegurar la inexistencia de una "Constitución británica” per se, que englobe a los diferentes Estados que forman el Reino Unido. Por el contrario, destaca la existencia de unos documentos de orden jurídico con una carga histórica tan importante que conforman en sí el sistema jurídico del país. Entre ellos, se pueden mencionar los siguientes documentos: La Carta Magna (forma denominativa también utilizada para la Constitución Española) fue redactada el 15 de junio de 1215 durante el reinado de Juan I ("Juan sin tierra") e igualmente ratificada por Eduardo I en 1297. Sin duda, podemos considerarla piedra angular que determina un precedente en el sistema jurídico inglés.

Otro de los documentos esenciales dentro del common law es el Bill of Rights, cuyo origen tiene lugar por medio de la denominada "Glorious Revolution", primera revolución occidental a favor de los derechos en el mundo, 
Esta revista forma parte del acervo de la Biblioteca Jurídica Virtual del Instituto de Investigaciones Jurídicas de la UNAM

que tuvo lugar entre 1688 y 1689, la Act of Settlement 1701, una de las bases de la independencia judicial del common law en el mundo (Stevens, 2005: 1). La Union 1707 Act, por medio del cual se sella la unión de la corona escocesa con la inglesa, pasando a formar un único reino, denominado Gran Bretaña.

El siglo XX es testigo de gran cantidad de documentos emanados del Parlamento y que constituyen de igual modo la fuente del derecho inglés y, por tanto, del common law. Dentro del sistema parlamentario podemos citar las Parliament Acts 1911 y 1949 y la Life Peerage Act 1958. Asimismo, la House of Commons Disqualification Act 1975 excluye diversas categorías como futuros miembros del Parlamento, entre los que se encuentran Lords, funcionarios, miembros en activo de las fuerzas armadas o miembros pertenecientes a órdenes religiosas, por citar algunos ejemplos. En tercer lugar, la Supreme Court Act 1981, denominada más tarde como Senior Courts Act 1981, define la estructura del Tribunal Supremo del Reino Unido.

Dentro del siglo XXI podemos mencionar otros documentos legislativos de especial relevancia:

- Civil Contingencies Act 2004.

- Constitutional Reform Act 2005.

- Constitutional Reform and Governance Act 2010.

- Fixed-term Parliament Act 2011.

- Succession to the Crown Act 2013.

Por tanto, la inexistencia de un código constitucional escrito dentro del derecho inglés o common law es clave en la consiguiente divergencia en los tres subsistemas judiciales británicos, puesto que, junto con sus diversos contextos históricos diferenciadores, han hecho que el sistema judicial británico posea tres subsistemas claramente diferenciados.

\section{El precedente. Stare decisis}

El sistema del common law se basa en dos vertientes fundamentales. Por un lado, las leyes que han sido aprobadas por el Parlamento del país en cuestión, gracias a las cuales podemos trazar la evolución de la jurisprudencia de su Estado. Por otra parte, los propios tribunales, así como 
las decisiones de éstos en forma de sentencias son esenciales en el sistema jurídico del common law.

En este sentido, la propia decisión del caso en litigio no es importante únicamente en tal caso, sino que contribuye a futuras decisiones tomadas en otros ulteriores. Así, por tanto, y como se ha mencionado anteriormente, el aspecto más notable del derecho inglés es la ausencia de un derecho codificado y, en especial, de un código civil, a favor del uso del precedente.

Por consiguiente, y a diferencia del papel fundamental del propio legislador, que codifica las normas jurídicas de un determinado Estado, el common law o derecho consuetudinario pasa a ser un sistema jurídico con abundante conocimiento práctico, basado en resolver posibles conflictos dentro de los propios tribunales. Nuevamente, la falta de una codificación escrita, distintiva del derecho romano y del derecho español (civil law), pone en relevancia el aspecto práctico del common law ante uno más codificado en el civil law.

El derecho inglés, principal sistema jurídico dentro del common law, basa su sistema del precedente en la máxima latina "stare decisis et non quieta movere", y que normalmente se abrevia en la forma "stare decisis". De este modo, la base del precedente reside en la certeza y el concepto de "justicia" en la ley, en la que no existe un único texto en el common law, sino una recopilación de sentencias que abarcan un periodo que puede extenderse durante más de dos siglos. Ahumada realiza una acertada comparación del llamado "stare decisis", propio de los tribunales del common law, con un "laboratorio de derecho": "el derecho se pone a prueba, se desarrolla y se transforma a impulso de conflictos reales que reclaman solución" (Ahumada, 2003: 353).

El "stare decisis" del common law se basa, por tanto, en el carácter práctico de los diferentes casos juzgados en el common law. Ante esta situación, el jurista del common law se encuentra ante un sistema menos férreo y carente de rigidez, en donde prima la decisión del judge, basada en casos con situaciones jurídicas similares juzgado en ese momento; un judge de manera individual, considerando los precedentes, dicta un fallo. Durante siglos los judges se han situado en la primacía de un razonamiento jurídico que, en palabras de Sir William Blackstone, pasaron a ser los "oráculos vivientes" del derecho: "En verdad los jueces hacen y cambian el derecho. La totalidad del common law es creación del juez y, sólo mediante la modificación 
judicial en el derecho, el common law conserva su vigencia en un mundo cambiante" (Whittaker, 2006: 38).

La tesis de Hale y Blackstone es básicamente que el common law estaría constituido por usos y costumbres que han gobernado a los ingleses desde tiempos inmemorables, apoyados en los principios generales de justicia entre los particulares y la conveniencia pública, que se puede modificar por medio de una ley emanada del Parlamento (Cross et al., 2012: 49).

El ordenamiento jurídico inglés, del mismo modo que otros provenientes del mismo, basa su derecho en un origen procesal o case law, por el cual el judge resuelve un caso teniendo en consideración aquello que se ha fallado en casos precedentes. Dentro del precedente, Ashworth (2000) define el "guideline precedent" del siguiente modo:

A guideline judgment is a single judgment which sets out general parameters for dealing with several variations of a certain type of offence, considering the main aggravating and mitigating factors, and suggesting an appropriate starting point or range of sentences. This kind of judgment was pioneered in the 1970s by Lawton P.J.... (Ashworth, 2000: 30).

Busby et al. identifican del mismo modo las ventajas del precedente, coincidiendo con Ahumada en la consistencia y justicia que emana del "stare decisis" del common law: "Without a system of precedent, the law would be more uncertain and it is likely that more court actions would be brought in the face of conflicting views on the state of the law" (Busby et al., 2008: 38).

Cownie et al. (2010) recalcan la importancia de los judges que dictan sentencias que serán consideradas por otros judges. No obstante, debemos tener en cuenta ciertas consideraciones. En primer lugar, podemos deducir del precedente la inmediatez en el fallo del caso, puesto que el judge únicamente tiene que recurrir a casos precedentes con el objetivo de poder basar en ellos su fallo.

Por ello, no es necesaria la consulta de la legislación a fin de poder basar tal fallo, al contrario que en derecho romano, napoleónico o el denominado en el Reino Unido como derecho continental, en el que todo se encuentra totalmente tipificado en códigos en los que se basa a la hora de aplicar el derecho.

Dentro del precedente, se pueden considerar los siguientes criterios:

Esta obra está bajo una Licencia Creative Commons

Atribución-NoComercial-SinDerivar 4.0 Internacional, IIJ-UNAM.

Boletín Mexicano de Derecho Comparado, núm. 154, enero-abril de 2019, pp. 513-538. 
- Se debe acatar toda decisión tomada en tribunales superiores dentro de la jerarquía judicial británica.

- Dichas decisiones tomadas por los tribunales superiores constituyen un precedente persuasivo para los tribunales de mayor jerarquía.

- Una decisión constituye siempre un precedente para los tribunales de inferior jerarquía respecto del tribunal que previamente ha dictado dicha sentencia.

Finalmente, si estas decisiones expresadas mediante los fallos de las sentencias forman el precedente para otros casos de características similares, todo ello puede influir en gran medida en el fallo del judge. Sin embargo, los fallos judiciales no se basan únicamente en la decisión de otros judges, sino que entran en escena otras características propias del common law: ratio decidendi y obiter dicta.

\section{Ratio decidendi y obiter dicta}

Otros de los puntos a tener en consideración dentro del common law es la distinción entre ratio decidendi y obiter dicta. En primer lugar, la ratio decidendi se puede definir como la opinión necesaria para el fallo final de la sentencia, mientras que las obiter dicta se pueden considerar como las opiniones adicionales aportadas en cada sentencia.

El judge inglés expone los motivos de su fallo, resume cuáles fueron los medios de prueba aportados, justificando del mismo modo las decisiones previas y sintetizando los argumentos expuestos por parte de los letrados de ambas partes. Tanto en las sentencias penales como en las civiles se realiza un resumen de los hechos, se sintetizan los argumentos presentados y se examinan cuestiones jurídicas relevantes.

Por este motivo, la ratio decidendi es vinculante para cualquier Court o tribunal, que debe seguir tanto si está de acuerdo o no, mientras que las obiter dicta no son vinculantes, si bien es cierto que pueden tener cierto valor en el fallo de la sentencia. Pueden ser persuasivas y su valor es capital, pues han sido consideradas por jueces, todo ello importante para sentencias venideras.

En cualquier caso, la ratio decidendi concierne especialmente a los casos en los cuales no se expresan las razones de la decisión tomada, aportándose una enumeración de los hechos, con una síntesis de los argumentos 
presentados por las partes. En la actualidad los tribunales superiores expresan las razones de su decisión, siendo improbable su inclusión en los repertorios de jurisprudencia (law reports) si no lo hacen.

La argumentación en las sentencias británicas constituye pieza clave dentro de las mismas. Dada su falta de codificación, las sentencias británicas incluyen un desglose detallado de todos los aspectos a considerar en las mismas con el objetivo de fijar aquellos aspectos legislativos que sientan precedentes en otras sentencias de tribunales inferiores.

El obiter dictum se puede definir como "una proposición jurídica contenida en el voto del juez que desde el punto de vista lógico no puede ser considerada la premisa mayor en relación con los hechos selectos de la decisión" (Cross y Harris, 2012: 100).

Dentro de una sentencia podemos observar más de una ratio decidendi, teniendo cada una de ellas su propia importancia y siendo vinculante de acuerdo con las reglas relativas a la jerarquía de los Courts. Si se presentan varios votos razonados que llegan a una misma conclusión, pero no están de acuerdo en la ratio, surge cierta dificultad y arbitrariedad.

Cross y Harris (2012) consideran el hecho primordial del common law: el judge está obligado, a la hora de dictar sentencia, a tener en consideración casos previos como material en el que basar su decisión en su propio caso.

El precedente en el common law tiene los siguientes puntos a tener en cuenta:

- Todos los tribunals están obligados a seguir y continuar con el precedente que se haya originado en cualquier tribunal superior.

- No se tienen en consideración las sentencias anteriores en su totalidad, puesto que en ciertas ocasiones no hay sentencias similares. Sí se tienen en cuenta las ratio decidendi, siendo, en palabras de Cross y Harris (2012: 27), concisas y dogmáticas.

\section{Actus reus $y$ mens rea}

En primer lugar, mencionaremos el "actus reus", denominado "guilty $a c t$ " y cuyo concepto es definido por Cross del siguiente modo: "The external behaviour or conduct which is prohibited by the Criminal law... It also includes a range of the other behaviour requirements, defined in each

Esta obra está bajo una Licencia Creative Commons

Atribución-NoComercial-SinDerivar 4.0 Internacional, IIJ-UNAM.

Boletín Mexicano de Derecho Comparado, núm. 154, enero-abril de 2019, pp. 513-538. 
criminal offence. For example, the actus reus of theft is taking someone else's property (Cross, 2011: 16)".

Herring (2011) define igualmente el concepto de "actus reus" por medio de las cuatro "C", y que se resumen del siguiente modo:

- Conducta (conduct), puesto que el actus reus requiere un comportamiento ilegal.

- Circunstancias (circumstances), dicho comportamiento tiene lugar en un escenario determinado.

- Contexto (context), de tipo interno y un "estado mental" que hace que dicho comportamiento suponga un delito penal.

- Consecuencias (consequences), que producen un resultado ilegal por medio del comportamiento.

Sin embargo, el "actus reus" de un delito no es únicamente una cuestión de conducta por parte del encausado, puesto que, como apunta Herring: "The term «actus reus» can include a state of affairs for which the accused is responsable, such as having an offensive weapon in a public place... or being in the possession of a controlled drug..." (Herring, 2011: 40).

En procesos penales se tiene en cuenta que el delito es la combinación de mens rea y actus reus, no siendo suficiente el mens rea para considerarse delito, si bien es cierto que no siempre es de este modo. Del mismo modo, una persona puede ser encausada y condenada por no realizar una acción obligada (acción frente a omisión), esto es, por la omisión más que el acto en sí. Por otro lado, el actus reus puede tener en sí mismo un elemento de carácter mental, el de la propia víctima, como es el caso de la falta de consentimiento por parte de la víctima de una agresión sexual.

En segundo lugar, podemos considerar el término "mens rea" o "guilty mind", que se puede definir como todo pensamiento interno que tiene que estar presente en la mente de alguien en el momento de cometer la acción delictiva, o "actus reus" (premeditación). De este modo, el encausado pasa a ser un condenado por un delito penal, puesto que el mens rea se refiere a la culpabilidad judicial, no moral.

Sin embargo, y del mismo modo que ocurría en el actus reus, no siempre se requiere que haya mens rea para que se produzca un delito. Tal es el caso de la conducción temeraria, o de todo acto que requiera un riesgo 
innecesario, dando lugar a un actus reus (culpa vs. dolo). Cross (2011) distingue dos tipos de riesgos temerarios para tener en cuenta:

- Subjective recklessness (o temeridad subjetiva), en la que el encausado predijo cierto riesgo en el actus reus, si bien es cierto que asumió dicho riesgo, causando un actus reus.

- Objective recklessness, en el que se habría precedido un riesgo grave y obvio del actus reus como resultado del riesgo que él mismo asumió (Cross, 2011: 36).

\section{DERECHO INGLÉS Y GALÉS}

Los apartados precedentes nos han presentado los rasgos sobresalientes del common law, case law o derecho consuetudinario. Sin embargo, dicho ordenamiento, propio de Inglaterra, Gales y demás países angloparlantes, no surgió independientemente de la sociedad y del contexto histórico en el que se enmarca. Por ello, recordemos un breve compendio histórico de Inglaterra y Gales con el claro objetivo de poder atisbar el origen del derecho consuetudinario y su posterior evolución.

Por ello, plantearemos un marco teórico del derecho inglés y galés con el objetivo de poder trazar la evolución histórica de dicho ordenamiento y, de este modo, comprender la idiosincrasia del ordenamiento jurídico de Inglaterra y Gales.

\section{ORIGEN Y EVOLUCIÓN DEL DEREGHO INGLÉS Y GALÉS}

Una vez explicados los rasgos generales de Inglaterra y Gales, pasamos a abordar someramente el origen de su ordenamiento jurídico.

Ajani et al. (2010) afirman que el origen del derecho inglés es milenario y establecen el origen del ordenamiento jurídico inglés y galés en la época normanda, momento en el que las tropas provenientes del norte de Francia invadieron las islas en 1066, guiadas por Guillermo el Conquistador. Según apunta Glenn (2007), la llegada de los normandos a Inglaterra no era más que un fenómeno normal en su expansión hacia el exterior, con la consiguiente reconciliación con las normas que en ese momento existían en tierras inglesas. 
Sin embargo, el ordenamiento jurídico normando, introducido por Guillermo el Conquistador no fue el primero originado en las islas. El primer caso del que se tiene conocimiento tuvo lugar hacia el año 85 d. C., y los primeros textos que han llegado hasta nosotros son las recopilaciones normativas redactadas sobre el año 600 d. C., denominadas "Laws of Wihtred"' (cerca de 700 d. C.).

Mientras tanto, Gales mantuvo una legislación no escrita hasta el siglo XII, haciendo uso del denominado "triadic verse form", comenzando cierta codificación a partir de escritos de abogados bajo el reinado del rey galés Hywel Dda (942-950). En 1258 Llewelyn ap Gruffudd se proclamó príncipe de Gales. Los writs ingleses se introdujeron formalmente en Gales en 1284, el mismo año en el que se proclamó el Statute of 1284, por el que Gales no formaba parte del reino inglés y que reforzaba las costumbres galesas. No obstante, el reino inglés comenzó a extender su influencia hacia Gales, sobre todo en lo concerniente a la administración del terreno.

En 1536 se otorgó a los súbditos galeses los mismos derechos y libertades que a los ingleses, si bien es cierto que Gales continuó manteniendo sus organismos propios. De ese modo, podemos constatar la creación del Great Session en Gales, un nuevo sistema de Tribunal Supremo galés, en 1541. Sin embargo, con el paso de los siglos, ambos reinos comenzaron a unificarse, dando lugar a la abolición de los Great Sessions galeses en 1830, aunando Gales e Inglaterra en un único territorio.

Por consiguiente, el derecho inglés y galés comparten un solo sistema judicial, denominado "derecho inglés" y que comparte, como hemos expuesto, diversos puntos convergentes en todos los sistemas judiciales del common law, tanto dentro del Reino Unido como en otros territorios, y cuyos detalles se mencionan en las siguientes secciones.

\section{DERECHO GIVIL Y DERECHO PENAL BRITÁNICO}

La rama del derecho civil dentro del ordenamiento del common law resuelve disputas entre personas físicas o entre personas físicas y el Estado.

La principal normativa del derecho civil es el Crown Procedure Rules (CPR). Por medio de la Prosecution of Offenders Act 1985 se estableció el Crown Prosecution Service (CPS), dirigido por el Director of Public Prosecution (DPP) y cuyo origen se remonta a los denominados "Public Prosecutions", 
órgano propio a finales del siglo XIX. El DPP se encuentra, asimismo, bajo la supervisión del Attorney General, miembro del gabinete que responde de su gestión ante el Parlamento, siendo el CPS la figura procesal que domina la acusación en la actualidad.

En ese momento, Inglaterra y Gales se dividieron en áreas, cada una de las cuales con un Chief Crown Prosecutor, cuya responsabilidad recae en supervisar las operaciones del CPS. Dicha figura jurídica sigue un procedimiento por medio de diversos documentos, entre los que podemos nombrar, por su importancia, el Code for Crown Prosecutors, que se publica anualmente.

Por su parte, dentro del derecho penal debemos considerar la Law Commission, definido del siguiente modo:

A body [that] considers the codification of the law, the elimination of anomalies, the repeal of obsolete and unnecessary enactments, a reduction in the number of separate enactments, and simplification and modernization generally. The Commission consists of a chairman and four other members, appointed by the Lord Chancellor from among the holders of judicial office, barristers, solicitors, and academic lawyers. There is a separate Commission for Scotland (Oxford University, 2015: 358).

El derecho inglés dispone asimismo de la Law Commission Act 1965, gracias a la cual se establecen unas pautas con el fin de poder redactar una legislación clara y en constante revisión para evitar cualquier tipo de ambigüedad. Dicha ley estipula en su artículo 3o. (1): “to take and keep under review all the law... with a view to its systematic development and reform", siendo su práctica habitual la producción de un "consultation paper", por el que las personas interesadas pueden informarse.

El derecho penal castiga la conducta que no puede causar daños, pero puede hacer peligrar la vida de otros (como es el caso de la conducción temeraria), que puede desencadenar una infracción penal. Es por tanto, que para ello debemos considerar el concepto de "delito" (crime), de acuerdo con el Oxford Dictionary of Law: "The act (or sometimes the failure to act) that is deemed by statute or by the Common Law to be a public wrong and is therefore punishable by the state in criminal proceeding" (Oxford University, 2015: 161).

Simester et al. definen igualmente el concepto de "crime": "A crime is an event that is prohibited by law, one which can be followed by a prose-

Esta obra está bajo una Licencia Creative Commons

Atribución-NoComercial-SinDerivar 4.0 Internacional, IIJ-UNAM.

Boletín Mexicano de Derecho Comparado, núm. 154, enero-abril de 2019, pp. 513-538. 
cution in criminal proceedings and, thereafter, by punishment on conviction. A crime must be defined by reference to the legal consequences of the act" (Simester et al. 2013: 1).

La Law Commission ha estado trabajando en la redacción de un Código Penal durante las últimas décadas, tras su primer borrador de éste en 1985, aunque abandonó el proyecto en 2008. Dado que no existe un Código Penal para Inglaterra y Gales, la tipificación de los delitos proviene de una legislación determinada que trata diversos tipos de hechos delictivos.

Los delitos penales se juzgan tanto en los magistrates' court como los crown courts, atendiendo a la naturaleza de los delitos. Dichos actos son considerados moralmente erróneos y merecen un castigo, si bien es cierto que no siempre ocurre de ese modo.

Herring (2011) y Padfield (2012) realizan una clasificación de los delitos en varios grupos.

- Indictable offences, triable by a jury in a crown court. Delitos graves o muy graves, juzgados en el crown court, con un juicio con jurado y tras la resolución adoptada por un magistrates' court.

- Summary offences (triable by magistrates' court). Delitos menos graves, juzgados por un procedimiento inmediato y abreviado en los magistrates' courts.

- Triable either-way offences. Delitos que son enjuiciables por cualquiera de los dos courts mencionados.

\section{ORIGEN Y EVOLUGIÓN DEL DERECHO ESCOCÉS}

El derecho escocés es otro de los sistemas jurídicos con una larguísima trayectoria y que forma parte de los tres subsistemas judiciales británicos a estudio. El derecho escocés cuenta con su propio carácter distintivo frente al de otros ordenamientos jurídicos, como es el inglés, dada su dilatada historia paralela a la historia de su vecino inglés, manteniendo su propio carácter distintivo tras la Act of Union, momento en el que se proclamó la adhesión a Inglaterra en 1707. En ese momento, se unifican los Parlamentos de Escocia e Inglaterra, si bien mantiene este último ciertas divergencias con sus leyes y sistemas jurídicos independientes. 
Busby et al. (2008) apuntan cómo las guerras por la independencia entre Inglaterra y Escocia marcaron, aún más, las divergencias de ambos países y, del mismo modo, sus ordenamientos jurídicos. Mientras que el derecho inglés se mantuvo fiel al common law, en el caso del derecho escocés éste fue trasladándose al marco del denominado derecho romano.

Puesto que el derecho escocés se ha desarrollado independientemente del inglés, los courts escoceses han establecido sus propios principios e incluso han incorporado algunos de sus delitos penales cambiándoles el nombre, como es el caso de "culpable homicide" en Escocia, frente a "offence of manslaughter", su correspondiente en derecho inglés.

El Parlamento escocés consta de 129 miembros que se reúnen en Edinburgo, 73 de los cuales son provenientes de las "constituencies" o circunscripciones electorales, mientras que los otros provienen de 8 regiones electorales.

El sistema judicial escocés constituye, por tanto, un subsistema dentro del sistema judicial británico con numerosas convergencias y divergencias que merecen su estudio dentro del derecho comparado, hecho que merece detallar en los siguientes apartados.

\section{Derecho GIVIL y DeReGho PenAl escocéS}

$\mathrm{Al}$ igual que el sistema jurídico inglés, el sistema escocés se divide en dos grandes ramas: por una parte, aquellos casos en los que se juzgan litigios entre personas físicas y jurídicas. Por ejemplo, en el caso de desalojos ("evictions") o divorcios ("divorce"). Por otro lado, están los casos contra individuos en casos graves como agresión sexual ("rape") o violencia física ("assault").

Estos casos civiles se pueden tratar en los Sheriff Courts, quienes abordan litigios tales como separación o divorcio, custodia, adopción o incumplimientos de contrato, entre otros.

Por otro lado, el Criminal Law, definido como: "a body of rules designed to ensure the peaceful coexistence of citizens within the community" (Busby et al., 2008: 20). En el derecho escocés no se encuentra tipificado en un único código, sino que se encuentra recogido mediante una combinación de delitos.

El derecho penal escocés se distingue de otros sistemas judiciales británicos al establecer tres posibles veredictos en los litigios penales: "guilty", que se define como: "culpable o responsable de un delito. Veredicto entre-

Esta obra está bajo una Licencia Creative Commons

Atribución-NoComercial-SinDerivar 4.0 Internacional, IIJ-UNAM.

Boletín Mexicano de Derecho Comparado, núm. 154, enero-abril de 2019, pp. 513-538. 
gado al finalizar un proceso penal contra quien ha sido hallado culpable de un hecho delictivo" (Ramos y Gleeson, 2014: 98). Asimismo, encontramos el veredicto "not guilty", como no "culpable, inocente". Por otro lado, el derecho escocés, a diferencia del derecho inglés, incorpora un nuevo veredicto, "proven", veredicto de "sin faltas" o "de falta de pruebas", (Alcaraz y Hughes, 2007: 458)

Hay tres courts escoceses que dictan sentencias penales:

- The High Court of Justiciary. Para casos más graves, entre los que se incluyen la agresión sexual y el asesinato.

- The Sheriff Court. Dicta sentencias para otros casos penales. Son casos que se tratan por medio del "solemn procedure" y del "summary procedure".

- The Fustice of the Peace.

\section{ORIGEN Y EVOLUCIÓN DEL DERECHO NORIRLANDÉS}

Irlanda del Norte es el tercer sistema jurídico británico a tener en cuenta dentro del sistema judicial británico. La historia de Irlanda del Norte (Northern Ireland o "Tuaisceart Éireann" en irlandés) viene marcada por su conflicto de carácter religioso y político con Inglaterra entre católicos y protestantes, cuyo origen se remonta al siglo XVII. Tras algunos siglos de pugna, en 1921 se creó Irlanda del Norte (Ulster), tras unificarse los condados de Antrim, Armagh, Cavan, Donegal, Down, Fermanagh, Derry o Londonderry, Monaghan y Tyrone, separándose del resto de la República de Irlanda.

En Irlanda del Norte, una de las figuras jurídicas clave en el proceso penal es el "Public Prosecution Service for Northern Ireland" (PPS) que a modo de acusación formal funciona como un órgano independiente tanto de la policía como del propio gobierno, al igual que ocurre en Inglaterra y Gales. Asimismo, el PPS informa al encausado sobre el órgano judicial que va a juzgar su caso, y se pone en contacto con este con el fin de fijar una fecha para el juicio, así como estudiar la disponibilidad del acusado.

De la misma manera que en el caso del sistema judicial inglés, el PPS debe llevar a un individuo a juicio, si bien es cierto que tal caso debe de ser probado "beyond a reasonable doubt", es decir, no se puede considerar al acusado culpable hasta que no se recopilen los elementos probatorios ("evidence") para luego poder formalizar la acusación de tal individuo. 
El sistema judicial norirlandés comparte cierta similitud con el inglés, pudiendo constatar una mayor diferenciación entre los sistemas de Inglaterra y Gales y el sistema de Escocia dentro del ámbito jurídico. No obstante, a pesar de las divergencias evidentes entre los tres subsistemas británicos, la divergencia es más acusada entre el common law y el civil law español, del que pasamos a mencionar sus rasgos más sobresalientes a continuación.

\section{DERECHO ESPAÑOL}

El derecho civil (civil law), también denominado "derecho romano" o "derecho napoleónico", es la segunda familia de derecho a estudio. El término "civil law" deriva de la expresión latina "ius civile", por la que los romanos designaban a aquellas leyes en privilegio de los ciudadanos romanos, frente al resto de los ciudadanos, que tenían el "ius gentium".

Glenn (2007) afirma que la noción de "ius gentium" tenía dos vertientes. Por una parte, la ley que concernía a todo el mundo, incluyendo a los propios romanos, aunque con pocas consecuencias. Por otro, existían unos preceptos que los romanos utilizaban con aquellos que no eran considerados ciudadanos. Es por ello, que el "ius gentium" se desarrolló de manera notable cuando la civilización romana comenzó su contacto con otros pueblos en su expansión por el mundo.

El mismo autor define al derecho romano del siguiente modo: "Roman law... is famous for its precision, detail, and apparent timelessness... Roman law was the law of an empire... The Romans took what turned out to be a unique view of the legal relations of the world, and it has never been followed since..." (Glenn, 2007: 2).

\section{Fuentes del derecho español}

El derecho español se basa en las siguientes fuentes:

a) La ley. La ley se contrapone frente a la costumbre, puesto que mientras que la ley es escrita y cierta, la costumbre es un derecho no escrito y, por consiguiente, incierto (García y Fernandez-Galiano, 1996: 92).

Esta obra está bajo una Licencia Creative Commons

Atribución-NoComercial-SinDerivar 4.0 Internacional, IIJ-UNAM.

Boletín Mexicano de Derecho Comparado, núm. 154, enero-abril de 2019, pp. 513-538. 
b) La costumbre. Se puede definir como un consentimiento tácito y unánime por parte de un determinado pueblo, arraigado dentro de su sistema social.

c) Asimismo, la doctrina jurídica atiende a dos razones básicas, gracias a las cuales podemos hablar de costumbre jurídica: por una parte, el "usus", elemento externo o repetición general y uniforme, constante, de cierta conducta. Por otro lado, el elemento interno o la conciencia de obligatoriedad del mismo.

d) Los principios generales del derecho. Son los enunciados normativos más generales utilizados por los jueces y legisladores, así como los juristas en general. Ejemplos: equidad, libertad, justicia, fraternidad, igualdad, etcétera.

e) La jurisprudencia. Por medio de códigos que, a diferencia del common law, muestran la jurisprudencia tipificada por escrito.

Asimismo, mencionamos la existencia de diversas fuentes esenciales de derecho español:

- La Constitución de 1978: representa la norma jurídica básica del derecho español. La Constitución establece la norma suprema que articula el orden político e incluso incorpora otras fuentes de derecho, aparte de ella misma.

- Las leyes orgánicas. Recogidas en el artículo 81 de la Constitución, versan sobre diversas materias, como los derechos fundamentales, las libertades públicas, la aprobación de leyes autonómicas, etcétera.

- Las leyes ordinarias. Emanadas de las Cortes, existen dos tipos de leyes: leyes del pleno del Congreso y leyes de comisión, a partir de una comisión determinada que la aprueba.

- Los decretos-legislativos. Dictados por el gobierno, dichos decretos emanan del Poder Ejecutivo a partir de la legislación (Poder Legislativo).

- Los decretos-ley. Los dicta el gobierno con carácter de urgencia (“con fuerza de ley"), a diferencia del decreto-legislativo, dictados por las Cortes. Dado su carácter de urgencia, el decreto-ley no afecta a materias estipuladas en el título I de la Constitución española, con relación a los derechos y deberes de la ciudadanía. 
- Los códigos: el derecho español se encuentra tipificado mediante diversos códigos, como son el Código Civil, Penal, Contencioso-Administrativo, etcétera.

- El ordenamiento de las comunidades autónomas. Entes con autonomía a partir del Estado descrito en la Constitución española de 1978. Así, podemos mencionar los Estatutos de Autonomía, fundamento de las 17 comunidades autonómas del país.

- El BOE (Boletín Oficial del Estado) es el diario oficial encargado de la publicación de la legislación, decretos y ordenamientos de España. Así, citaremos otras fuentes del derecho español, como los reglamentos parlamentarios, tratados internacionales y el derecho comunitario.

\section{DERECHO COMPARADO}

El derecho comparado se puede considerar un efecto propio de la sociedad globalizada de los siglos XX y XXI. Si bien es cierto que anteriormente destacaron otros momentos históricos en los que las culturas se han relacionado entre sí, la relación entre los diversos países se ha incrementado exponencialmente con relación a los avances en las comunicaciones y los negocios en el mundo, siendo su resurgimiento en los últimos veinticinco años a partir de la globalización.

El origen del derecho comparado es bastante reciente y se puede fechar a partir del primer congreso internacional de legislación comparada, celebrada en la exposición mundial en París en el año 1900. Más adelante, la Segunda Guerra Mundial hizo que el derecho comparado surgiera con más fuerza en Europa y Estados Unidos, en un intento de buscar posibles modos de convivencia jurídica en común.

Dicha disciplina atiende a diversas denominaciones, como "legislación comparada" o "ciencia comparada del derecho", dentro de la doctrina alemana, "teoría general comparada del derecho", por la doctrina inglesa, o bien "derecho contrastado", término defendido por algunos juristas hispanohablantes. Finalmente hemos optado por "derecho comparado" puesto que es una terminología que engloba, en mayor o menor medida, a todas las mencionadas, siendo la más empleada en la península.

Édouard Lambert y Raymond Saleilles coinciden en la necesidad de poder establecer un derecho común para la humanidad con el objetivo

Esta obra está bajo una Licencia Creative Commons

Atribución-NoComercial-SinDerivar 4.0 Internacional, IIJ-UNAM.

Boletín Mexicano de Derecho Comparado, núm. 154, enero-abril de 2019, pp. 513-538. 
de poder eliminar posibles divergencias de los distintos sistemas jurídicos. Ante esta situación, el derecho comparado surge con el objetivo de hacer una comparativa de las diferentes familias del derecho, anteriormente comentadas en detalle, con el fin de poder vislumbrar puntos en común y divergencias en los distintos sistemas jurídicos de los distintos países, llegando a haber un entendimiento entre ellos.

Sin embargo, la primera y segunda guerras mundiales fueron el detonante de un debilitamiento más que notable de esta idea de una "ley mundial". Sin embargo, gracias al derecho comparado podemos crear ciertos paralelismos existentes entre numerosos sistemas jurídicos pertenecientes o no a la misma familia del derecho. Del mismo modo, podemos observar las diferencias en sus ordenamientos jurídicos, figuras legales, órganos judiciales y demás organismos que forman parte del propio sistema del país.

Otra de las particularidades del derecho comparado es la comparación que los profesionales del derecho, es decir, juristas o expertos en derecho, pueden realizar acerca de los diferentes sistemas jurídicos a analizar. Instituciones, tribunales, jueces, son analizados y contrastados en dicha disciplina con el fin de poder cotejarse concienzudamente. El derecho comparado, una de las partes esenciales del presente capítulo, mejora y amplía el conocimiento, tanto de los ordenamientos jurídicos de los países en cuestión, como del ordenamiento propio de nuestra nación, haciendo posible que incluso se puedan resolver conflictos de carácter jurídico y lingüístico.

Es en este último punto en el que se ha realizado la comparación entre ambos sistemas jurídicos. Por un lado, el sistema jurídico británico, compuesto de tres subsistemas claramente distinguibles que han merecido su estudio en profundidad con el claro objetivo de establecer una comparativa entre ellos.

Los tres subsistemas jurídicos distinguen diversos aspectos a tener en consideración dentro del derecho comparado: tribunales, jueces y otros conceptos jurídicos distinguibles pasan a formar parte de las divergencias entre los tres subsistemas jurídicos británicos. Por otro lado, el derecho español con sus particularidades diferentes del common law, analizado igualmente en el estudio. 


\section{Conclusiones}

Finalmente mostraremos una serie de aspectos a tener en cuenta. En primer lugar, hemos realizado una distinción entre el derecho consuetudinario o common law y el derecho romano, civil o civil law. Dicha diferencia se basa en un conjunto de características que son aspectos distintivos de ambas familias del derecho.

Dentro de dicho compendio entre los países a estudio y sus ordenamientos jurídicos, se han establecido de igual modo diversos puntos a tener en cuenta. En primer lugar, se han desglosado las diversas ramas del derecho que podemos encontrar en ambas familias, esto es, derecho consuetudinario y derecho civil y las subáreas más representativas.

En esta situación observamos la familia romano-germánica, de marcado carácter codificado frente a la familia consuetudinaria, cuya codificación es mucho menos influyente, fortaleciendo en gran medida el concepto del "precedente". Tamarit (2007) resalta, a modo de ejemplo, los ordenamientos jurídicos de España e Inglaterra y Gales. En el caso de España, Tamarit afirma su carácter rígido, con un catálogo de circunstancias agravantes y atenuantes, en este caso con motivo de la codificación del ordenamiento jurídico español (Tamarit, 2007: 31-33).

Por otro lado, están Inglaterra y Gales, donde tiene especial importancia la pena de multa o sanción, que concede importancia a las penas monetarias impuestas por los tribunales frente a las penas de prisión.

No podemos, en este punto, obviar las conclusiones de Fletcher (1997), quien afirma:

En el mundo jurídico de habla española es quizás el derecho angloamericano, llamado tradicionalmente common law, una de las culturas jurídicas de los países occidentales menos conocidos. Ello se debe... a las peculiaridades y "rarezas" que dicho sistema tiene para nuestra sensibilidad jurídica... y a veces desprecio, que frente a él ha mostrado siempre nuestros más grandes juristas, muchos más atentos... a las grandes construcciones que a partir del siglo XIX empezaron a producirse en otros países europeos... como Francia, luego Italia y finalmente Alemania... (Fletcher, 1997: 11).

El presente estudio ha contribuido a establecer un resumen de los parámetros comparativos entre los tres sistemas jurídicos del Reino Unido con el sistema jurídico español con el objetivo de compilar aspectos de

Esta obra está bajo una Licencia Creative Commons

Atribución-NoComercial-SinDerivar 4.0 Internacional, IIJ-UNAM.

Boletín Mexicano de Derecho Comparado, núm. 154, enero-abril de 2019, pp. 513-538. 
ambos para investigadores que quieran realizar una consulta de las características fundamentales de ambos ordenamientos jurídicos, a partir de su origen y evolución histórica.

Por consiguiente, uno de los objetivos del presente trabajo es la relación bidireccional de ambos sistemas judiciales. De este modo, se facilita el trasvase de información y equivalencias entre conceptos jurídicos, enmarcados en una cultura e historia determinada que no se puede dejar de lado, pieza fundamental en los ordenamientos jurídicos incluidos en dos sistemas heterogéneos analizados, common law y civil law.

\section{BIBLIOGRAFÍA}

OxFORD UnIVERSITy, 2015, Oxford Dictionary of Law, Oxford University Press.

ADAME GODDARD, Jorge, 2017, ¿Qué es el matrimonio? Su naturaleza ética y jurídica, México, UNAM, Instituto de Investigaciones Jurídicas.

Aguiló Regla, Josep, 2000, Teoría general de las fuentes del derecho y del orden jurídico), Barcelona, Ariel.

AHumadA RuIZ, María Ángeles, 2003, "Stare decisis y creación judicial de derecho constitucional. A propósito del precedente constitucional en el sistema judicial norteamericano de Ana Laura Magaloni Kerpel”, Revista Española de Derecho Constitucional, 23.

AJANI, Gianmaria et al., 2010, Sistemas jurídicos comparados, lecciones y materiales, Barcelona, Universitat.

Alcaraz Varo, Enrique y Hughes, Brian, 2007, Diccionario de Términos Furídicos, Madrid, Ariel.

Alcaraz Varo, Enrique y Hughes, Brian, 2009, El españoljurídico, Ariel. Alcaraz Varo, Enrique, 2002, El inglés jurídico, Madrid, Ariel.

Ashworth, Andrew, 2000, Sentencing and Criminal Justice, Law in Context, London, Butterworths,

ASPREY, Michele, 1991, Plain Language for Lawyers, Annandale, Federation Press.

BAKER, John Hamilton, 2002, An Introduction to English Legal History, Butterworths.

Bernal Del GaStillo, Jesús, 201 1, Derecho penal comparado. La definición del delito en los sitemas anglosajón y continental, Barcelona, Atelier.

Bolinger, Dwight, 1975, Aspects of Language, Harcourt Brace Jovanovich. 
Esta revista forma parte del acervo de la Biblioteca Jurídica Virtual del Instituto de Investigaciones Jurídicas de la UNAM

BorJa, Anabel, 2000, El texto jurídico inglés y su traducción al español, Barcelona, Ariel.

BORJA, Anabel, 2007, Estrategias, materiales y recursos para la traducción juridical inglés-español, Barcelona, Ariel.

BROOK, George Leslie, 1973, Varieties of English, London, MacMillan,

BusBy, Nicole et al., 2008, Scots Law, A Student's Guide, Edinburgh, Tottel Publishing.

Cownie, Fiona et al., 2007, English Legal System in Context, Oxford University Press.

Cross, Rupert y HARRIS, James, 2012, El precedente en el derecho inglés, Madrid, Barcelona y Buenos Aires, Marcial Pons.

Cross, Noel, 2011, Criminal Law and Criminal Fustice, an Introduction, Londres, Sage.

D’ors, Xavier, 2001, Antología de textos jurídicos de Roma, Tres Cantos, Akal.

DAINOw, Joseph, 1996, "The Civil Law and the Common Law, Some Points of Comparison", The American Fournal of Comparative Law (ASCL) 15.

Damian Moreno, Juan, 2002, Introducción al sistema judicial español, Pamplona, Aranzadi.

Damian Moreno, Juan, 2014, La decisión de acusar. Un estudio a la luz del sistema acusatorio inglés, Pamplona, Dykinson.

Doran, Sean y Jackson, John (eds.), 2000, The Fudicial Role in Criminal Proceedings, Oxford.

Dressler, Joshua, 2006, Understanding Criminal Law, Newark, Lexis Nexis.

ESMEIN, Adhémar, 1905, "Le droit comparé et l'enseignement du droit", Congrés International de Droit Compare.

Fernandez Martínez, Juan Manuel, 2012, Diccionario Furídico, Pamplona, Thomson Reuters Aranzadi.

FLeTCHeR, George, 1997, Conceptos básicos de derecho penal, Valencia, Tirant lo Blanch.

Garcia Garrido, Manuel y Fernandez-Galiano, Antonio, 1996, Iniciación al derecho, Madrid, Universitas.

Garofalo, Giovanni, 2009, Géneros discursivos de la justicia penal. Un análisis contrastivo español-italiano orientado a la traducción, Milano, Franco Angeli.

Glenn, H., Patrick, 2007, On Common Laws, Oxford, Oxford University Press.

Gomez DE LiaÑo, Fernando, 1992, El proceso penal. Tratamiento jurisprudencial, Oviedo, Forum.

Esta obra está bajo una Licencia Creative Commons

Atribución-NoComercial-SinDerivar 4.0 Internacional, IIJ-UNAM.

Boletín Mexicano de Derecho Comparado, núm. 154, enero-abril de 2019, pp. 513-538. 
GotTI, Maurizio, 2008, Investigating Specialized Discourse, Bern, Peter Lang. Heller, Kevin y Dubber, Markus, 2011, The Handbook of Comparative Criminal Law, Standford, Stanford Law Books.

Herring, Jonathan, 2011, Criminal Law, Basingstoke-New York, Padgrave MacMillan.

Hertel, Christian, 2009, "Sistemas y familias jurídicos del mundo", Sistemas jurídicos del mundo, Notarius International.

KeARns, Martin, 2007, Legal English, Madrid, Colex.

López Samaniego, Anna, 2010, "Documentos judiciales con destinatarios no expertos. El empleo de los mecanismos referenciales en la sentencia del 11M", Revista Signos, 43.

MaEr, Lucinda y GAY, Oonagh, 2009, The Bill of Rights 1689, House of Commons Library.

MORAN, Gloria M., 2002, "El derecho comparado como disciplina jurídica, la importancia de investigación y la docencia del derecho comparado y la utilidad del método comparado en el ámbito jurídico", Anuario da Facultade de Dereito da Universidade Coruña.

Ormeron, David, 2008, Smith and Hogan Criminal Law, Oxford, Oxford University Press.

Padfield, Nicola, 2012, Criminal Law, Oxford, Oxford University Press.

Ramos Bossini, Fernando y Gleeson, Mary, 2014, Diccionario Bilingüe de Terminología Legal, Granada, Comares.

SAULA, Joan, 2013, El abogado en casa, Barcelona, De Vecchi.

Simester, Andrew et al., 2013, Simester and Sullivan's Criminal Law, Theory and Doctrine, Oxford, Hart Publishing.

SINDER, Janet, 2001, Irish Legal History, An Overview and Guide to the Sources, Law Library Journal.

SLAPPER, Gary y KelLy, David, 2015, The English Legal System (20152016), Londres, Routledge.

Stevens, Robert, 2005, The English Fudges, Their Role in the Changing Constitution, Oxford, Hart Publishing.

STOLzE, Radegundis, 2013, "The Legal Translator's Approach to Texts", Humanities.

TAMARIT SumaLla, Josep María, 2007, "Sistema de sanciones y política criminal. Un estudio de derecho comparado europeo", Revista Electrónica de Ciencia Penaly Criminología. 
Esta revista forma parte del acervo de la Biblioteca Jurídica Virtual del Instituto de Investigaciones Jurídicas de la UNAM

TARANILla, Raquel, 2015, "El empleo de imágenes en la sentencia judicial, (reflexiones sobre la evolución del género)", Revista Llengua i Dret.

Tiersma, Peter, 1999, Legal Language, Chicago, The University of Chicago Press.

VÁZQUEZ Y DEL ARBOL, Esther, 2008, La traducción (inglés-español) de testamentos británicos y documentos relacionados. De la teoría a la práctica, Granada, Universidad.

VÁZQUEZ Y DEL ARBOL, Esther,2014, Derecho civil comparado aplicado a la traducción jurídico-judicial (Reino Unido y España), Madrid, Dykinson.

VÁzQUEZ Y DEL ÁRBOL, Esther, 2016, Traducción judicial y policial (inglésespañol) y derecho comparado Court and Police Translation (English-Spanish) and Comparative Law, Madrid, Dykinson.

WhitTAKer, Simon, 2006, "El precedente en el derecho inglés, una visión desde la ciudadela", Revista Chilena de Derecho, 35.

ZwEIGERT, Konrad y KÖTZ, Hein, 1998. Introduction to Comparative Law, Oxford, Clarendom Press. 